UCRL-CR--105109

DE91 002667

\title{
A Rapid Relativistic Distorted Wave Approach for Calculating Cross Sections for Ionization of Highly Charged Ions
}

\author{
H. L. Zhang \\ D. H. Sampson \\ Pennsylvania State University
}

October 22, 1990

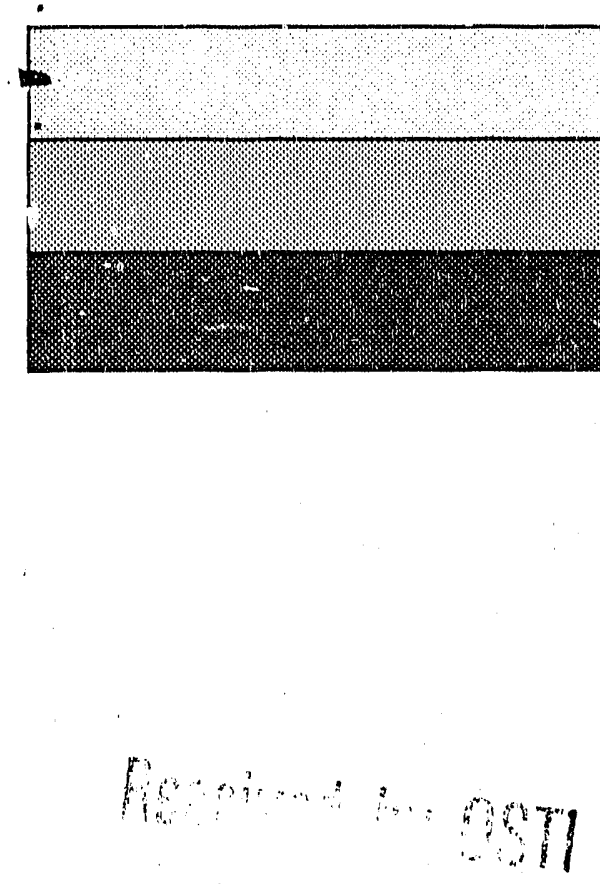

NOV 151990

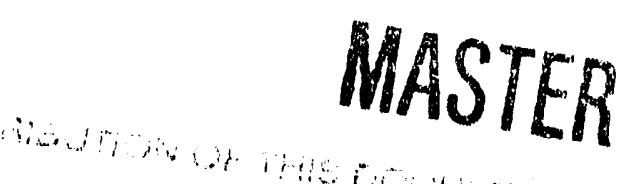


DISCLAIMER

Work performed under the auspices of the U.S. Department of Energy by lawrence Livermore Natisnal I aboratory under contract number W-7405-ENG-48.

This document was prepared as an account of work sponsored by an agency of the United States Government. Neither the United States Government nor the University of California nor any of their employees, makes any warranty, express or implieci, or assumes any legal liability or responsibility for the accuracy, completeness, or usefulness of any information, apparatus, product, or process disclosed, or represents that its use would not infringe privately owned rights. Reference herein to any specific commercial products, process, or service by trade name, trademark, manufacturer, or otherwise, does not necessarily constitute or imply its endorsement, recommendation, or favoring by the United States Goverument or the University of California. The views and opinions of authors expressed herein do not necessarily state or reflect those of the United States Government or the University of California, and shall not be used for advertising or product endorsement purposes. 


\title{
A Rapid Relativistic Distorted Wave Approach for Calculating Cross Sections for Ionization of Highly Charged Ions
}

\author{
Hong Lin Zhang and Douglas H. Sampson \\ Department of Astronomy, The Pennsylvania State University \\ University Park, Pennsylvania 16802
}

The rapid relativistic distorted wave method of Zhang et al [Phys. Rev. A $\underline{40}, 616$ (1989)] for excitation, which uses the atomic structure data of Sasıpson et al [Phys. Rev. A 40, 604 (1989)], has been extended to ionization. In this approach the same DiracFock-Slater potential evaluated using a single mean configuration is used in calculating the orbitals of all electrons bound and free. Values for the cross sections $Q$ for ionization of various ions have been calculated and generally good agreement is obtained with other recent relativistic calculations. When results are expressed in terms of the reduced ionization cross section $Q_{R}$, which is proportional to $I^{2} Q$, they are close to the non-relativistic Coulomb-Born-Exchange values of Moores et al [J. Phys. B $\underline{13}, 385$ (1080)] for hydrogenic ions except for high $Z$ and/or high energies. This suggests that fits of the $Q_{R}$ to simple functions of the impact electron energy in threshold units with coefficients that are quite slowly varying functions of an effective $Z$ can probably be made. This would be convenient for plasma modeling applications. 


\section{INTRODUCTION}

Highly charged ions with very large values for the nuclear charge number $Z$ are becoming of increased interest in the study of high temperature plasmas, partly due to the interest in developing ultrashort wave length lasers. For highly charged ions with $Z \gtrsim 25$ or 30 the $j$ dependence of the radial functions for some orbitals becomes significant so that a fully relativistic approach based on the Dirac equation should be used in calculating the properties of such ions. For the applications to high temperature plasma modeling it is also desirable to have a very rapid relativistic approach because an immense amount of atomic data is required. In references 1 and 2 such an approach was developed and in Ref. 3-6 it was applied to large scale production of collision strengths, or equivalently excitation cross sections, and oscillator strengths. As discussed in these references, the approach appears to be accurate for

$$
Z \gtrsim 2 N \text { or } 2.5 N
$$

where $N$ is the number of bound electrons per ion.

The approach was also recently extended to give cross sections for excitation of highly charged ions to specific magnetic sublevels by a directive beam of electrons. ${ }^{7}$ This was motivated by the need for such cross sections in the modeling and design of EBIT experiments at the Lawrence Livermore National Laboratory (LLNL) ${ }^{8-10}$ For this purpose ionization cross sections are needed, as well. Of course, ionization cross sections are also needed for the modeling of high temperature plasmas and recently electron-impact ionization cross sections for $\mathrm{U}^{91+}$ and $\mathrm{U}^{90+}$ have been measured. ${ }^{11}$ The purpose of the present work is to extend the approach of Ref. 1-7 to give relativistic electron-impact ionization cross sections. Since ionization is like excitation summed over many final levels, as seen by comparing Eqs (2) and (13) below, one expects the range of accuracy for ionization to be at least as great as for excitation given by $\mathrm{Eq}(1)$.

In the next section the theory used in the present work is described. Then in 
section III numerical results are given for ionization from the $1 s, 2 s, 2 p_{1 / 2}$ and $2 p_{3 / 2}$ subshells of various types of ions with various values of $Z$ and comparison is made with other recent works. ${ }^{12-16}$

\section{OUTLINE OF THEORY}

The present relativistic distorted wave ionization program was obtained by modification of the relativistic distorted wave excitation program of Ref. 2 . Hence we first briefly review the approach used for excitation. Then we indicate the modifications required for ionization. By combining Eqs (1) and (3) of Ref. 2, one can write the expression for the relativistic distorted wave cross section $Q(i-f)$ for the excitation transition $i-f$ in an $N$-electron ion in the form

$$
Q(i-f)=\frac{8 \pi a_{0}^{2}}{k^{2} g_{i}} \sum_{J}(2 J+1) \sum_{\kappa, \kappa^{\prime}}\left|\left\langle\Psi_{i}\left|\sum_{\substack{q, p \\ q<p}}^{N+1} \frac{1}{r_{q p}}\right| \Psi_{f}\right\rangle\right|^{2}
$$

Here $a_{\circ}$ is the Bohr radius, $k$ is the relativistic wave number of the impact electron, $g_{i}$ is the statistical weight of the initial level of the $N$-electron target ion and $\kappa$ and $\kappa^{\prime}$ are the initial and final relativistic angular momentum quantum numbers of the free electron. The $\Psi_{i}$ and $\Psi_{f}$ in $\mathrm{Eq}(2)$ are the initial and final antisymmetric wave functions for the total $(N+1)$-electron system consisting of target ion plus free electron.and $J$ is the total angular momentum quantum number for this entire system. The relation between $k$, the relativistic momentum $p$ and the kinetic energy $\epsilon$ of the impact electron is

$$
k^{2}=\frac{p^{2} a_{\circ}^{2}}{\hbar^{2}}=\epsilon\left[1+\frac{\alpha^{2}}{4} \epsilon\right]
$$

where $\alpha$ is the fine structure constant $e^{2} / \hbar c$ and $\epsilon$ is in Rydbergs. The $\kappa$ in Eq (2) is related to the orbital and total angular momentum quantum numbers $l$ and $j$ for the impact electron in the usual way

$$
\kappa=i, j=l-1 / 2 ; \quad \kappa=-(l+1), j=l+1 / 2
$$


Of course, analogous relations apply between $\kappa^{\prime}, l^{\prime}$ and $j^{\prime}$ pertaining to the scattered electron.

The initial antisymmetrized function $\Psi_{i}$ in Eq (2) can be written ${ }^{17}$

$$
\Psi_{i}=\frac{1}{(N+1)^{1 / 2}} \sum_{k=1}^{N+1}(-1)^{N+1-k} \sum_{M_{t}, m} C\left(J_{t} j M_{t} m ; J M\right) \Psi_{\beta_{t} J_{t}}\left(x_{k}^{-1}\right) u_{\epsilon l j m}\left(x_{k}\right)
$$

where $x_{k}$ designates the space and spin coordinates of electron $k$ and $x_{k}^{-1}$ means the space and spin coordinates for all $N$ electrons other than electron $k$. The $\Psi_{\beta_{i} J_{t}}\left(x_{k}^{-1}\right)$ is the initial antisymmetrized target ion wave function constructed of Dirac spinors or orbitals as in $\mathrm{Eq}(4)$ of Ref. 1. Here $J_{t}$ is the quantum number corresponding to the total initial angular momentum of the target ion and $\beta_{t}$ represents all other quantum numbers required to specify the initial state of the target ion. The $u_{\epsilon l j m}$ in Eq (5) is a Dirac spinor for the initial free electron (impact electron) in a central potential $V(r)$ due to the target ion. Specifically

$$
u_{\epsilon l j m}(x) \equiv u_{\epsilon \kappa m}(x)=\frac{1}{r}\left[\begin{array}{c}
P_{\epsilon \kappa}(r) \chi_{\kappa m}(\theta, \phi, \sigma) \\
i Q_{\epsilon \kappa}(r) \chi_{-\kappa m}(\theta, \phi, \sigma)
\end{array}\right]
$$

where the $\chi_{\kappa m}$ are the usual spin-angular momentum functions and the large and small components of the radial functions $P_{\epsilon \kappa}$ and $Q_{\epsilon \kappa}$ satisfy the coupled Dirac equations

$$
\left[\frac{d}{d r}+\frac{\kappa}{r}\right] P_{\epsilon \kappa}(r)=\frac{\alpha}{2}\left[\epsilon \quad V+\frac{4}{\alpha^{2}}\right] Q_{\epsilon \kappa}(r)
$$

and

$$
\left[\frac{d}{d r}-\frac{\kappa}{r}\right] Q_{\epsilon \kappa}(r)=-\frac{\alpha}{2}[\epsilon-V] P_{\epsilon \kappa}(r)
$$

Similar to $\mathrm{Eq}(5)$ the final function $\Psi_{f}$ in $\mathrm{Eq}(2)$ for the excitation cross sections is given by

$$
\Psi_{f}=\frac{1}{(N+1)^{1 / 2}} \sum_{k=1}^{N+1}(-1)^{N+1-k} \sum_{M_{t}^{\prime}, m^{\prime}} C\left(J_{t}^{\prime} j^{\prime} M_{t}^{\prime} m^{\prime} ; J M\right) \Psi_{\beta_{t}^{\prime} J_{t}^{\prime}}\left(x_{k}^{-1}\right) u_{\epsilon^{\prime} l^{\prime} j^{\prime} m^{\prime}}\left(x_{k}\right)
$$

where primed quantities pertain to the final state in the exactly analogous way that cor. responding unprimed quantities in Eq (5) pertain to the initial state. 
In order to extend $\mathrm{Eq}(2)$ to ionization all that is required is the following: (1) The $\Psi_{\beta_{t}^{\prime} J_{t}^{\prime}}\left(x_{k}^{-1}\right)$ in $\mathrm{Eq}_{\mathrm{q}}(9)$ must be replaced by an antisymmetrized wave function for an $N$-electron system corresponding to the final $(N-1)$-electron ion plus an ejected electron

$$
\Psi_{\beta_{t}^{\prime} J_{t}^{\prime}}\left(x_{k}^{-1}\right)=\frac{1}{N^{1 / 2}} \sum_{p \neq k}^{N+1}(-1)^{N-p} \sum_{M_{t}^{\prime \prime}, m^{\prime \prime}} C\left(J_{t}^{\prime \prime} j^{\prime \prime} M_{t}^{\prime \prime} m^{\prime \prime} ; J_{t}^{\prime} M_{t}^{\prime}\right) \Psi_{\beta_{t}^{\prime \prime} J_{t}^{\prime \prime}}\left(x_{p}^{-1}\right) u_{\epsilon^{\prime \prime} l^{\prime \prime} j^{\prime \prime} m^{\prime \prime}}\left(x_{p}\right)
$$

where $\Psi_{\beta_{t}^{\prime \prime} J_{t}^{\prime \prime}}$ is the antisymmetrized wave function corresponding to the final $(N-1)$-electron ion with total angular momentum $J_{t}^{\prime \prime}$ and $u_{\varepsilon^{\prime \prime}\left(\prime^{\prime \prime} j^{\prime \prime} m^{\prime \prime}\right.}\left(x_{p}\right)$ is a Dirac spinor for the ejected electron analogous to the Dirac spinor $u_{\epsilon l j m}$ given by Eqq. (6-8) for the impact electron. A consequence of this is that then $P_{n_{a}^{\prime} l_{a}^{\prime \prime} j_{a}^{\prime}}$ and $Q_{n_{a}^{\prime} l_{a}^{\prime} j_{a}^{\prime}}$ in the direct and exchange radial scattering matrix elements given by Eqs (9) and (10) of Ref 2 are replaced with $P_{\epsilon^{\prime \prime} l^{\prime \prime} j^{\prime \prime}}$ and $Q_{\epsilon^{\prime \prime} l^{\prime \prime} j^{\prime \prime}}$. (2) Eq (2) must be summed over the total final angular momentum $J_{t}^{\prime}$ for the system consisting of the $(N-1)$-electron final ion with total angular momentum $J_{t}^{\prime \prime}$ plus the ejected electron with total angular momentum $j^{\prime \prime}$. (3) Eq (2) must also be summed over $\kappa^{\prime \prime}$ or equivalently $j^{\prime \prime}$ and $l^{\prime \prime}$ for the ejected electron. (4) Eq (2) must be integrated over the range 0 to $(\epsilon-I) / 2$ for the energy $\epsilon^{\prime \prime}$ of the ejected electron, where $I$ is the ionization energy. (5) Finally one must divide by a factor of $\pi$ to account for the fact that a final bound electron function with normalization

$$
\int_{0}^{\infty}\left[P_{n_{a}^{\prime} \kappa_{a}^{\prime}}^{2}(r)+Q_{n_{a}^{\prime} \kappa_{a}^{\prime}}^{2}(r)\right] d r=1
$$

has been replaced with a free ejected electron function with normalization

$$
\int_{0}^{\infty}\left[P_{\epsilon^{\prime \prime} \kappa^{\prime \prime}}(r) P_{\epsilon^{\prime \prime \prime} \kappa^{\prime \prime}}(r)+Q_{\epsilon^{\prime \prime} \kappa^{\prime \prime}}(r) Q_{\epsilon^{\prime \prime \prime} \kappa^{\prime \prime}}(r)\right] d r=\pi \delta\left(\epsilon^{\prime \prime}-\epsilon^{\prime \prime \prime}\right)
$$

In summary the relativistic distorted wave ionization cross section is given by

$$
Q=\frac{8 a_{o}^{2}}{k^{2} g_{i}} \sum_{J}(2 J+1) \sum_{J_{t}^{\prime}} \sum_{\kappa, \kappa^{\prime}, \kappa^{\prime \prime}} \int_{0}^{(\epsilon-I) / 2} d \epsilon^{\prime \prime}\left|\left\langle\Psi_{i}\left|\sum_{\substack{q, p \\ q<p}}^{N+1} \frac{1}{r_{q p}}\right| \Psi_{f}\right\rangle\right|^{2}
$$

with $\mathrm{Eq}_{\mathrm{q}}(10)$ applied to $\mathrm{Eq}(9)$ for $\Psi_{f}$. 
All the orbitals bound and free entering Eq (13) are Dirac spinors of the form given by Eq (6) with the radial functions satisfying equations of the form of Eqs (7) and (8). In fact, in the present approach the same central potential $V(r)$ is used for all electrons bound and free so the orbitals are all automatically orthogonal. This potential is the relativistic Hartree-Fock-Slater potential, or so-called Dirac-Fock-Slater potential given in Rydbergs by

$$
V(r)=-\frac{2 Z}{r}+V_{c}(r)-\left(\frac{24}{\pi} \rho\right)^{\frac{1}{3}}
$$

where

$$
V_{c}(r)=\sum_{n^{\prime} \kappa^{\prime}} w_{n^{\prime} \kappa^{\prime}} \int_{0}^{\infty} \frac{2}{r_{>}}\left[P_{n^{\prime} \kappa^{\prime}}^{2}\left(r_{2}\right)+Q_{n^{\prime} \kappa^{\prime}}^{2}\left(r_{2}\right)\right] d r_{2}
$$

and

$$
\rho(r)=\frac{1}{4 \pi r^{2}} \sum_{n^{\prime} \kappa^{\prime}} w_{n^{\prime} \kappa^{\prime}}\left[P_{n^{\prime} \kappa^{\prime}}^{2}(r)+Q_{n^{\prime} \kappa^{\prime}}^{2}(r)\right]
$$

Here $w_{n^{\prime} \kappa^{\prime}}$ is the occupation number of subshell $n^{\prime} \kappa^{\prime}=n^{\prime} l^{\prime} j^{\prime}$, the summation is over all occupied subshells, $r_{>}$is the greater of $r$ and $r_{2}$, and $P_{n^{\prime} \kappa^{\prime}}$ and $Q_{n^{\prime} \kappa^{\prime}}$ are the so-called large and small components of the radial function of an electron in the $n^{\prime} \kappa^{\prime}$ subshell. The su'vscript "a" used in $\mathrm{Eq}$ (11) to distinguish bound orbitals from free ones has been dropped here for convenience.

In the application to excitation in Ref. 3-6 the potential given by Eqs (14)-(16) was evaluated using a single mean configuration with fractional occupation numbers in which the occupation for the active electron was approximately split between initial and final shells. In obtaining the ionization results given in the next section we mostly used the initial configuration of the target ion in determining the potential with Eqs (14)(16). For example, in considering either innershell ionization or ionization of the valence electron of Li-like ions in the ground level the configuration $1 s^{2} 2 s$ was used, while for ionization of a $2 p_{1 / 2}$ electron in a Li-like ion the configuration $1 s^{2} 2 p_{1 / 2}$ was used. This is a simple, straightforward procedure. However, one that would more nearly correspond to the procedure used successfully for excitation in Ref. 3-7 would be to reduce the occupation 
number of the initial subshell of the active electron by 0.5 and put an occupation number of 0.5 in a very high subshell to mock up the effect of the ejected electron. Thus a few test cases, for which results in the next section are indicated by stars as superscripts, were done this way. Specifically, test cases were done using the mean configurations

$$
\begin{aligned}
& 1 s^{1.5} 2 s^{1} 6 d_{5 / 2}^{0.5}, \\
& 1 s^{2} 2 s^{0.5} 6 d_{5 / 2}^{0.5}, \\
& 1 s^{2} 2 p_{1 / 2}^{0.5} 6 d_{5 / 2}^{0.5},
\end{aligned}
$$

and

$$
1 s^{2} 2 s^{2} 2 p_{1 / 2}^{1.5} 2 p_{3 / 2}^{4} 6 d_{5 / 2}^{0.5}
$$

in determining the potential with Eqs (14)-(16) for innershell ionization of Li-like ions in the ground configuration, ionization of the valence electron in Li-like ions initially in the $1 s^{2} 2 s$ and $1 s^{2} 2 p_{1 / 2}$ configurations, and ionization of a $2 p_{1 / 2}$ electron in neon-like ions in the ground configuration, respectively. These altered potentials affect results appreciably only for relatively low $Z$, where the electron-electron contribution to the potential is most significant.

Although we expect eventually to write a more general program, at present the computer program only calculates ionization cross sections with the form of hydrogenic cross sections except that the orbitals are calculated using the potential of Eqs (14)-(16). Thus, in this case $J_{t}^{\prime \prime}=0$ and $J_{t}^{\prime}=j^{\prime \prime}$ so the summation over $J_{t}^{\prime}$ is omitted. The present program can obviously be applied to ionization of the valence electron in Li-like, Na-like and $\mathrm{Cu}$-like ions. However, as shown in the Appendix of Ref. 18, a program such as the present one has much wider applications. In particular it applies whenever both the initial and final states are pure states, such as is the case for ionization of He-like, Ne-like and $\mathrm{Ni}$-like ions in their ground levels, and it also applies if only either the initial or final level is a pure state. Hence, it is applicable for ionization of F-like and Co-like ions as well 
as innershell ionization of $\mathrm{Li}$-like, $\mathrm{Na}$-like and $\mathrm{Cu}$-like ions. In order to make application to these more complex cases one must multiply by the initial occupation number $w_{n \kappa}$ of the active subshell $n \kappa$ and, if more than one state for the final ion is possible, one must multiply by a branching ratio factor $R$ considered, for example, in Ref. 19. Also if mixing occurs in the initial or final level, one must multiply by the square of the mixing coefficient and sum over the mixed states. It is also convenient to express results in terms of a reduced cross section $Q_{R}$ by factoring out a $\pi a_{\circ}^{2} / I(R y d)^{2}$ factor, where $I$ is the ionization energy. Then, if both initial and final states are pure ones, the cross section for ionization from subshell $n \kappa$ is given by

$$
Q=\frac{\pi a_{\circ}^{2}}{I(R y d)^{2}} w_{n \kappa} R Q_{R}
$$

while, if either the initial or final state is a mixed one, Eq (21) should be multiplied by the square of the mixing coefficient and summed over the mixed states, as mentioned previously.

Finally, we note that it is well known that the relative phase of the two final free electrons is unknown when the central field approximation has been made in determining their orbitals. The choice of phase used in our approach is what is sometimes called the "natural"- phase approximation, see Eq (10) of Ref. 14. This is the correct choice in the spccial case $\mathrm{cf}_{\mathrm{i}}$ a non-relativistic treatment as $Z \rightarrow \infty$. Thus, one might expect it is to be a good approximation for highly charged ions.

\section{NUMERICAL RESULTS AND DISCUSSION}

Ionization cross sections by the present method are compared with relativistic results available by other methods in Table I. The entries labeled Ref. 16 are results calculated with the relativistic distorted wave program used in the calculations of the direct ionization contribution, as compared with the indirect excitation-autoionization contribution, in Ref. 15 dealing with ionization of Na-like $\mathrm{Au}(Z=79)$. That program also uses the so-called "natural"-phase approximation, Eq (10) of Ref. 14. Thus, it differs from the 
present program in the physics used only in that the bound, incident, scattered and ejected electron functions are calculated in the Dirac-Fock potential ${ }^{20}$ rather than the more approximate Dirac-Fock-Slater potential used here. This is seen to have little effect in the cases considered in Table I, especially for the more highly charged ions, where the nuclear potential more completely dominates. The results in Ref. 12 and 13 were obtained in a similar way to those of Ref. 16 except that the "maximum inteference"-phase approximation [see Eq (11) of Ref. 14] was used. Of course, this gives smaller cross sections than the "natural"-phase approximation, but the difference is usially small. One sees that the present results are also close to those of Ref. 12 and 13, but are always larger, as expected. Finally, the results of Ref. 14 differ from the others in that they include the full lowestorder QED interaction between the electrons rather than simply the Coulomb interaction $1 / r_{i j}$ in calculating the scattering amplitudes. In other words they include the so-called generalized Breit interaction. However, they omit the exchange and interference terms, which they estimate to have no more than a $15 \%$ effect. Thus, in comparing with those results we also omit these terms. The agreement is seen to be rather good in this case as well, which is consistent with the conclusion reached in Ref. 14 and also demonstrated for hydrogenic ions in Ref. 21 that inclusion of the generalized Breit interaction has little effect on ionization until high impact electron energies $\gtrsim 250 \mathrm{keV}$ are reached. Our results, like the relativistic calculations of these other workers, are about a factor of 4 smaller than the recent measurements of Ref. 11.

In Table II we give the present results for many additional cases involving ionization from the $1 s, 2 s, 2 p_{1 / 2}$ and $2 p_{3 / 2}$ subshells. In the interest of brevity closed inactive subshells are omitted in giving the transitions for neon-like ions. The results are given for the reduced ionization cross section $Q_{R}$, which is related to the ionization cross section according to $\mathrm{Eq}(21)$. In these cases the branching ratio $R$ is unity except for innershell ionization of Li-like ions, where it is $1 / 4$ and $3 / 4$ for ionization to the $(1 s 2 s)_{0}$ and $(1 s 2 s)_{1}$ states of the He-like ions, respectively. Results of Moores et al ${ }^{22}$ for the non-relativistic 
Coulomb Born-Exchange reduced ionization cross section $Q_{R}^{H}$ for hydrogenic ions in the limit $Z \rightarrow \infty$ are included for comparison. These are independent of $Z$.

It is interesting to note that comparisons with other more elaborate calculations and experiment made, for example, in Ref. 22 and 23 indicate the $Q_{R}^{H}$ lead to cross sections that are quite accurate for $Z$ satisfying $\mathrm{Eq}_{\text {(1) }}$ with $Z \leq 26$. This tends to confirm our expectation that the present relativistic distorted wave results are accurate for $\mathrm{Z}$ satisfying Eq (1) because one sees from Table II that the present resu'ts agree rather well with the corresponding $Q_{R}^{H}$ values for low and intermediate $Z$. Unfortunately there are no results by the more elaborate relativistic programs of Ref. 12-16 with which we can compare for large $Z$ barely satisfying Eq (1). However, the comparisons for Na-like and Ne-like selenium $(Z=34)$ ions made in Table I with the results of Ref. 16 do show good agreement for $Z \simeq 3 N$ and $Z=3.4 N$, which are quite close to $Z=2 N$ or $2.5 N$.

Initially all our calculations were done using the initial configuration of the target ion in dete mining the Dirac-Fock-Slater potential with Eqs (14)-(16). However, some of the results for $Q_{R}$ for low $Z$, especially for ionization from the $2 p_{1 / 2}$ and $2 p_{3 / 2}$ subshells, looked slightly anomalous to us. Hence, we decided to do some additional test cases using the configurations given by Eqs (17)-(20) in determining the potential with Eqs (14)-(16). This latter procedure is more nearly like that used for excitation in Ref. 3-7. These results are indicated by stars as superscripts in Table II. One sees that the effect is quite large for $Z=8$, especially for ionization of the $2 p_{1 / 2}$ electron in Li-like ions in the $1 s^{2} 2 p_{1 / 2}$ configuration, ${ }^{24}$ where the starred entries are about $10 \%$ lower than corresponding unstarred entries. However, the effect rapidly decreases as $Z$ increases and is seen to be almost negligible $(\sim 2.5 \%)$ for the same transition when $Z=26$.

It appears that use of results determined using Eqs (17)-(20) would reduce the "bumpiness" in the data for low $Z$ and would give values for $Q_{R}$ for any given subshell that vary quite smoothly with ionization energy and for which quite simple fits could be made. In this connection we note that accurate fits of the $Q_{R}^{H}$ to simple functions of the 
impact electron energy in threshold units $u$ that are readily integrated over a Maxwellian electron distribucion function to obtain ionization rates have been made in Ref. 22. Since, as noted previously, the present relativistic results for $Q_{R}$ are generally quite close to those of Ref. 22 for $Q_{R}^{H}$ except for high $Z$ and/or high impact electron energies, one would expect that fairly accurate fits of the relativistic results for the reduced cross section could be made, as well, probably using the same functional form given by Eq (6) of Ref. 22, but allowing the coefficients to be slowly varying functions of an effective $Z$ or of $Z$ and $N$. This would be very convenient for applications to plasma" modeling.

In future work we will attempt to do this. Also we will consider ionization from additional higher subshells in various types of ions. In addition we expect to extend the approach to autoionization so that we can treat the excitation-autoionization contribution, which sornetimes considerably exceeds the direct contribution to ionization even for high $Z$, as shown in Ref. 15 .

\section{ACKNOWLEDGMENTS}

We wish to thank David L. Moores and Michael S. Pindzola for providing results prior to publication. This work was supported in part by the Innovative Science and Technology Office of the Strategic Defense Inttiative Organization under Contract N0001488-K-2021 and by the U.S. Department of Energy under Lawrence Livermore National Laboratory Subcontract 6181405 and Grant DE-FG02-85ER53208. 


\section{REFERENCES AND FOOTNOTES}

1. D. H. Sampson, H. L. Zhang, A. K. Mohanty and R. E. H. Clark, Phys. Rev. A $\underline{40}$, 604 (1989).

2. H. L. Zhang, D. H. Sampson and A. K. Mohanty, Phys. Rev. A 40 616 (1989).

3. H. L. Zhang and D. H. Sampson, At. Data Nucl. Data Tables $\underline{43}, 1$ (1989).

4. H. L. Zhang, D. H. Sampson and C. J. Fontes, At. Data Nucl. Data Tables $\underline{44}, 31$. (1990).

5. D. H. Sampson, H. L. Zhang and C. J. Fontes, At. Data Nucl. Data Tables $\underline{44} 209$ (1990).

6. H. L. Zhang, D. H. Sampson and C. J. Fontes, At. Data Nucl. Data Tables $\underline{44}, 273$ (1990).

7. H. L. Zhang, D. H. Sampson and R. E. H. Clark, Phys. Rev. A $\underline{41} 198$ (1990).

8. R.E. Marrs, M.A. Levine, D.A. Knapp and J.R. Henderson, Phys. Rev. Lett. $\underline{60}, 1715$ (1988).

9. R. E. Marrs, Bull. Am. Phys. Soc. 푸, 1391 (1989).

10. D. A. Knapp, C. L. Bennett, J. R. Henderson, R. E. Marrs and M. B. Schneider, Bull. Am. Phys. Soc. $\underline{34}, 1394$ (1989).

11. N. Claytor, B. Feinberg, H. Gould, C. E. Bemis, Jr, J. Gomez del Campo, C. A. Ludemann and C. R. Vane, Phys. Rev. Lett. 61, 2081 (1988).

12. M. S. Pindzola and M. J. Buie, ỉhys. Rev. A $\underline{37}, 3232$ (1988).

13. M. S. Pindzola and M. J. Buie, Phys. Rev. A $\underline{39}, 1029$ (1989).

14. M. S. Pindzola, D. L. Moores and D. C. Griffin, Phys. Rev. A $\underline{40}, 4941$ (1989).

15. K. J. Reed, M. H. Chen and D. L. Moores, Phys. Rev. A 41 , 550 (1990).

16. D. L. Moores and M. S. Pindzola, Phys. Rev."A (submitted).

17. The upper limit on the summation in the analogous $\mathrm{Eq}(5)$. of Ref. 2 should also be $N+1$ rather than $N$.

18. D.H. Sampson and H. L. Zhang, Phys. Rev. A $\underline{36}, 3590$ (1987). 
19. D.H. Sampson, Phys. Rev. A $\underline{34}, 986$ (1986).

20. I. P. Grant, B. J. McKenzie, P. H. Norrington, D. F. Mayers and N. C. Pyper, Compıt. Phys. Commun. 21, 207 (1980).

21. D. L. Moores and M. S. Pindzola, Phys. Rev. A $\underline{41}, 3603$ (1990).

22. D.L. Moores, L.B. Golden and D.H. Sampson, J. Phys. B $\underline{13}, 385$ (1980).

23. D.H. Sampson and L. B. Golden, J. Phys. B 11, 541 (1978).

24. Of couse, almost identical effects would occur at such low $Z$ if the $2 p_{1 / 2}$ electron were replaced with a $2 p_{3 / 2}$ electron. 


\section{TABLE CAPTIONS}

TABLE I. Comparison between present results and recent relativistic calculations by other workers of the cross sections $\left(\mathrm{cm}^{2}\right)$ for ionization from various sublevels of various types of icns with various values for the nuclear charge number $Z$. Here $2 \mathrm{p}^{*}$ means $2 \mathrm{p}_{1 / 2}$ and $2 p$ means $2 p_{3 / 2}$. The present results were obtained using the initial configuration of the target ion in calculating the Dirac-Fock-Slater potential with Eqs (14)-(16).

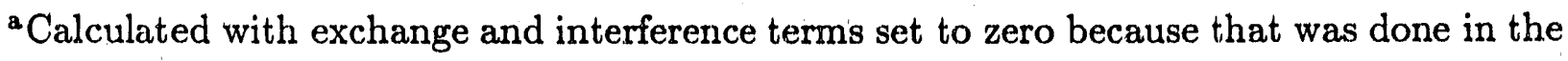
calculations of Ref. 14 .

TABLE II. Comparison of values for the reduced ionization cross section $Q_{R}$. Here $2 p^{*}$ means $2 p_{1 / 2}$ and $2 p$ means $2 p_{3 / 2}$. Unstarred entries were obtained using the initial configuration of the target ion in determining the Dirac-Fock-Slater potential with Eqs (14)-(16), while the starred entries were obtained using the configurations of Eqs (17)--(20) in determining the potential.

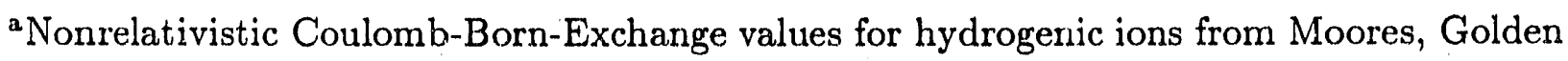
and Sampson, Ref. 22. These values are independent of $Z$. 


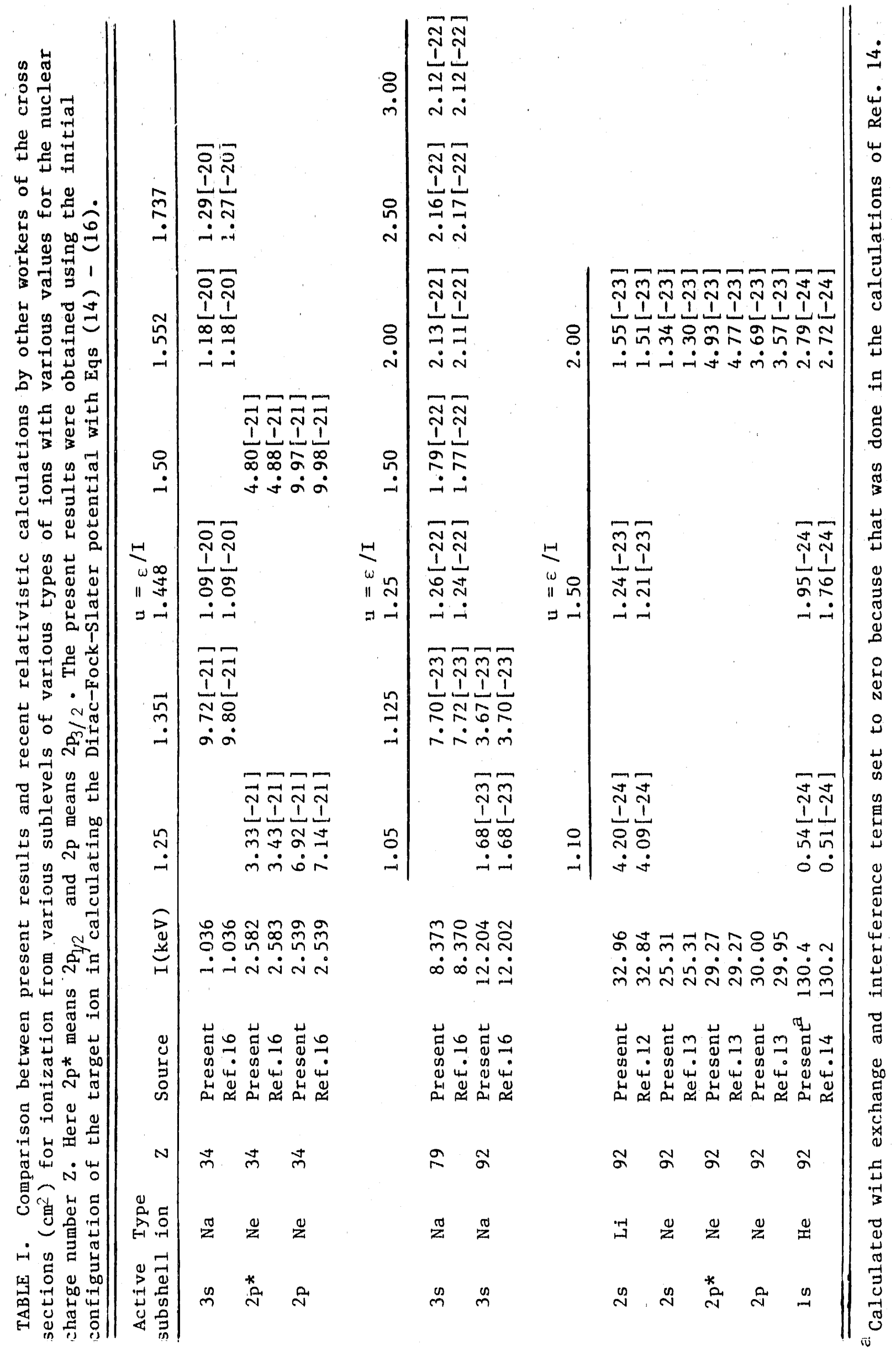


TABLE II. Comparison of values for the reduced fonization cross section $Q_{R}$. Here $2 p^{*}$ means $2 \mathrm{p}_{1} / 2$ and $2 \mathrm{p}$ means $2 \mathrm{p}_{3} / 2$. Unstarred entries were obtained using the initial configuration of the target ion in determining the Dirac-Fock-Slater potential with Eqs (14) - (16), while the starred entries were obtalned using the configurations of Eqs (17) - (20) in determining the potential.

\begin{tabular}{|c|c|c|c|c|c|c|c|c|c|c|}
\hline & $\begin{array}{l}\text { Type } \\
\text { ion }\end{array}$ & Transition & $Z$ & $I(R y)$ & 1.125 & 1.25 & $\begin{array}{l}u=\varepsilon / I \\
1.50\end{array}$ & 2.25 & 4.00 & 6.00 \\
\hline is & $\begin{array}{l}\mathrm{H}^{\mathrm{a}} \\
\mathrm{LI} \\
\mathrm{LI} \\
\mathrm{LI} \\
\mathrm{He} \\
\mathrm{LI} \\
\mathrm{LI} \\
\mathrm{He} \\
\mathrm{LI} \\
\mathrm{LI} \\
\mathrm{He} \\
\mathrm{LI} \\
\mathrm{LI} \\
\mathrm{He}\end{array}$ & $\begin{array}{l}1 s 22 s-(1 s 2 s) 1 \\
1 s 22 s-(1 s 2 s) 0 \\
1 s 22 s-(1 s 2 s) 0 \\
1 s 2-1 s \\
1 s 22 s-(1 s 2 s) 1 \\
1 s 22 s-(1 s 2 s) 0 \\
1 s 2-1 s \\
1 s 22 s-(1 s 2 s) 1 \\
1 s 22 s-(1 s 2 s) 0 \\
1 s 2-1 s \\
1 s 22 s-(1 s 2 s) 1 \\
1 s 22 s-(1 s 2 s) 0 \\
1 s 2-1 s\end{array}$ & $\begin{array}{r}8 \\
8 \\
8 \\
8 \\
26 \\
26 \\
26 \\
56 \\
56 \\
56 \\
92 \\
92 \\
92\end{array}$ & $\begin{array}{l}5.1316[1] \\
5.1921[1] \\
5.1921[1] \\
5.4259[1] \\
6.3881[2] \\
6.4108[2] \\
6.4949[2] \\
3.1798[3] \\
3.1855[3] \\
3.2049[3] \\
9.5369[3] \\
9.5506[3] \\
9.5865[3]\end{array}$ & $\begin{array}{l}0.283 \\
0.2667 \\
0.2691 \\
0.2602 * \\
0.2745 \\
0.2848 \\
0.2857 \\
0.2866 \\
0.3086 \\
0.3091 \\
0.3097 \\
0.3770 \\
0.3774 \\
0.3781\end{array}$ & $\begin{array}{l}0.479 \\
0.4598 \\
0.4643 \\
0.4716 \\
0.4850 \\
0.4865 \\
0.4878 \\
0.5260 \\
0.5268 \\
0.5277 \\
0.6427 \\
0.6435 \\
0.6447\end{array}$ & $\begin{array}{l}0.724 \\
0.7151 \\
0.7226 \\
0.6858 * \\
0.7293 \\
0.7396 \\
0.7421 \\
0.7429 \\
0.8041 \\
0.8055 \\
0.8064 \\
0.9852 \\
0.9865 \\
0.9881\end{array}$ & $\begin{array}{l}0.978 \\
1.0224 \\
1.0344 \\
1.0303 \\
1.0227 \\
1.0264 \\
1.0245 \\
1.1346 \\
1.1368 \\
1.1369 \\
1.4205 \\
1.4226 \\
1.4240\end{array}$ & $\begin{array}{l}1.0486 \\
1.0403 \\
1.0444 \\
1.0393 \\
1.2429 \\
1.2456 \\
1.2447 \\
1.6817 \\
1.6851 \\
1.6855\end{array}$ & $\begin{array}{l}0.819 \\
0.9251 \\
0.9366 \\
0.8605 * \\
0.9139 \\
0.9269 \\
0.9305 \\
0.9249 \\
1.2103 \\
1.2130 \\
1.2121 \\
1.7634 \\
1.7684 \\
1.7684\end{array}$ \\
\hline $2 s$ & $\begin{array}{l}\mathrm{Ha}^{\mathrm{a}} \\
\mathrm{LI} \\
\mathrm{LI} \\
\mathrm{Ne} \\
\mathrm{Li} \\
\mathrm{Ne} \\
\mathrm{Ne} \\
\mathrm{LI} \\
\mathrm{Ne} \\
\mathrm{Ne} \\
\mathrm{LI}\end{array}$ & $\begin{array}{l}1 s 22 s-1 s 2 \\
1 s 22 s-1 s 2 \\
2 s 2-2 s \\
1 s 22 s-1 s 2 \\
2 s 2-2 s \\
2 s 2-2 s \\
1 s 22 s-1 s 2 \\
2 s 2-2 s \\
2 s 2-2 s \\
1 s 22 s-1 s 2\end{array}$ & $\begin{array}{r}8 \\
8 \\
26 \\
26 \\
34 \\
56 \\
56 \\
79 \\
92 \\
92\end{array}$ & $\begin{array}{l}1.0139[1] \\
1.0139[1] \\
1.0257[2] \\
1.5042[2] \\
2.0232[2] \\
6.6576[2] \\
7.8134[2] \\
1.5083[3] \\
2.2051[3] \\
2.4232[3]\end{array}$ & $\begin{array}{l}0.321 \\
0.3131 \\
0.2966 * \\
0.2949 \\
0.3198 \\
0.3028 \\
0.3126 \\
0.3242 \\
0.3207 \\
0.3278 \\
0.3372\end{array}$ & $\begin{array}{l}0.532 \\
0.5240 \\
0.4947 \\
0.5312 \\
0.5062 \\
0.5214 \\
0.5389 \\
0.5353 \\
0.5476 \\
0.5624\end{array}$ & $\begin{array}{l}0.771 \\
0.7743 \\
0.7308 * \\
0.7336 \\
0.7745 \\
0.7462 \\
0.7661 \\
0.7875 \\
0.7882 \\
0.8078 \\
0.8275\end{array}$ & $\begin{array}{l}0.953 \\
0.9970 \\
0.9528 \\
0.9720 \\
0.9589 \\
0.9808 \\
0.9973 \\
1.0196 \\
1.0524 \\
1.0745\end{array}$ & $\begin{array}{l}0.8908 \\
0.8800 \\
0.8900 \\
0.9204 \\
0.9292 \\
0.9889 \\
1.0449 \\
1.0680\end{array}$ & $\begin{array}{l}0.695 \\
0.7646 \\
0.7040 * \\
0.7491 \\
0.7327 \\
0.7501 \\
0.7924 \\
0.8011 \\
0.8890 \\
0.9596 \\
0.9862\end{array}$ \\
\hline & $\begin{array}{l}\mathrm{H}^{\mathrm{a}} \\
\mathrm{LI} \\
\mathrm{LI} \\
\mathrm{Ne} \\
\mathrm{He} \\
\mathrm{LI} \\
\mathrm{LI} \\
\mathrm{Ne} \\
\mathrm{Ne} \\
\mathrm{LI} \\
\mathrm{Ne} \\
\mathrm{Ne} \\
\mathrm{LI}\end{array}$ & $\begin{array}{l}1 s 22 p^{*}-1 s 2 \\
1 s 22 p^{*}-1 s 2 \\
2 p^{*} 2-2 p^{*} \\
2 p^{*} 2-2 p^{*} \\
1 s 22 p^{*}-1 s 2 \\
1 s 22 p^{*}-1 s 2 \\
2 p^{*} 2-2 p^{*} \\
2 p^{*} 2-2 p^{*} \\
1 s 22 p^{*}-1 s 2 \\
2 p^{*} 2-2 p^{*} \\
2 p^{*} 2-2 p^{*} \\
1 s 2 \quad 2 p^{*-1}-1 s 2\end{array}$ & $\begin{array}{r}8 \\
8 \\
26 \\
26 \\
26 \\
26 \\
34 \\
56 \\
56 \\
79 \\
92 \\
92\end{array}$ & $\begin{array}{l}9.2544[0] \\
9.2544[0] \\
9.3633[1] \\
9.3633[1] \\
1.4684[2] \\
1.4684[2] \\
1.8980[2] \\
6.4199[2] \\
7.7192[2] \\
1.4683[3] \\
2.1512[3] \\
2.3984[3]\end{array}$ & $\begin{array}{l}0.409 \\
0.4522 \\
0.4140 * \\
0.4077 \\
0.3955 * \\
0.4236 \\
0.4132 * \\
0.4128 \\
0.4196 \\
0.4269 \\
0.4292 \\
0.4357 \\
0.4460\end{array}$ & $\begin{array}{l}0.668 \\
0.7492 \\
0.6752 \\
0.6939 \\
0.6810 \\
0.6912 \\
0.7007 \\
0.7089 \\
0.7216 \\
0.7372\end{array}$ & $\begin{array}{l}0.949 \\
1.0780 \\
0.9826 * \\
0.9801 \\
0.9514 * \\
0.9894 \\
0.9659 * \\
0.9822 \\
0.9949 \\
1.0030 \\
1.0253 \\
1.0489 \\
1.0682\end{array}$ & $\begin{array}{l}1.135 \\
1.3268 \\
1.2230 \\
1.1945 \\
1.2123 \\
1.2255 \\
1.2239 \\
1.2812 \\
1.3270 \\
1.3461\end{array}$ & $\begin{array}{l}1.0875 \\
1.1112 \\
1.1023 \\
1.2031 \\
1.2801 \\
1.2986\end{array}$ & $\begin{array}{l}0.786 \\
0.9614 \\
0.8566 * \\
0.9189 \\
0.8832 * \\
0.8531 \\
0.8298 * \\
0.9021 \\
0.9410 \\
0.9338 \\
1.0603 \\
1.1579 \\
1.1813\end{array}$ \\
\hline $2 p$ & $\begin{array}{l}\mathrm{H}^{\mathrm{a}} \\
\mathrm{Li} \\
\mathrm{Ne} \\
\mathrm{Li} \\
\mathrm{Ne} \\
\mathrm{Ne} \\
\mathrm{Li} \\
\mathrm{Ne} \\
\mathrm{Ne} \\
\mathrm{Li}\end{array}$ & $\begin{array}{l}1 s 22 p-1 s 2 \\
2 p 4-2 p 3 \\
1 s 22 p-1 s 2 \\
2 p 4-2 p 3 \\
2 p 4-2 p 3 \\
1 s 22 p-1 s 2 \\
2 p 4-2 p 3 \\
2 p 4-2 p 3 \\
1 s 22 p-1 s 2\end{array}$ & $\begin{array}{r}8 \\
26 \\
26 \\
34 \\
56 \\
56 \\
79 \\
92 \\
92\end{array}$ & $\begin{array}{l}9.2490[0] \\
9.2677[1] \\
1.4563[2] \\
1.8660[2] \\
6.1268[2] \\
7.3934[2] \\
1.3284[3] \\
1.8603[3] \\
2.0876[3]\end{array}$ & $\begin{array}{l}0.409 \\
0.4554 \\
0.4097 \\
0.4248 \\
0.4157 \\
0.4265 \\
0.4324 \\
0.4435 \\
0.4566 \\
1) .4641\end{array}$ & $\begin{array}{l}0.668 \\
0.7494 \\
0.6782 \\
0.6956 \\
0.6853 \\
0.7012 \\
0.7086 \\
0.7295 \\
0.7519 \\
0.7630\end{array}$ & $\begin{array}{l}0.949 \\
1.0783 \\
0.9839 \\
0.9914 \\
0.9874 \\
1.0063 \\
1.0118 \\
1.0486 \\
1.0828 \\
1.0962\end{array}$ & $\begin{array}{l}1.135 \\
1.3270 \\
1.2263 \\
1.1959 \\
1.2162 \\
1.2332 \\
1.2289 \\
1.2950 \\
1.3457 \\
1.3579\end{array}$ & $\begin{array}{l}0.977 \\
1.1779 \\
1.1081 \\
1.0446 \\
1.0886 \\
1.1107 \\
1.1002 \\
1.1961 \\
1.2656 \\
1.2800\end{array}$ & $\begin{array}{l}0.786 \\
0.9614 \\
0.9192 \\
0.8526 \\
0.9016 \\
0.9360 \\
0.9273 \\
1.0413 \\
1.1317 \\
1.1483\end{array}$ \\
\hline
\end{tabular}

a Nonrelativistic Coulomb-Born-Exchange values for hydrogenic ions from Monres, Golden and Sampson, Ref. 22. These values are independent of $\%$. 
